\title{
COMPOSIÇÃO FLORÍSTICA DE UMA FORMAÇÃO PIONEIRA COM INFLUÊNCIA FLUVIAL EM BALSA NOVA, PR, BRASIL
}

\author{
Carina Kozera ${ }^{1}$, Yoshiko Saito Kuniyoshi ${ }^{2}$, Franklin Galvão ${ }^{3}$, Gustavo Ribas Curcio $^{4}$ \\ ${ }^{1}$ Bióloga, Dra ${ }^{\text {. }, ~ U F P R, ~ C u r i t i b a, ~ P R, ~ B r a s i l ~-~ k o z e r a 23 @ y a h o o . c o m ~}$ \\ ${ }^{2}$ Naturalista, Dra ${ }^{\mathrm{a}}$, Depto. de Ciências Florestais, UFPR, Curitiba, PR, Brasil - yoshiko@ufpr.br \\ ${ }^{3}$ Eng. Florestal, Dr., Depto. de Ciências Florestais, UFPR, Curitiba, PR, Brasil - fgalvao@ufpr.br \\ ${ }^{4}$ Eng. Agrônomo, Dr., EMBRAPA Florestas, Colombo, PR, Brasil - curcio@cnpf.embrapa.br \\ Recebido para publicação: 18/08/2008 - Aceito para publicação: 18/09/2008
}

\begin{abstract}
Resumo
Com o objetivo de conhecer a composição florística da vegetação sobre uma planície de inundação do rio Iguaçu e incrementar coleções de herbários, foi realizado um levantamento florístico de uma Formação Pioneira com Influência Fluvial (várzea) em uma área de 4,4 ha em Balsa Nova, PR. Para tanto, foram efetuadas observações quinzenais e coletas de material para herborização e determinação. Foram encontradas 176 espécies, 103 gêneros e 46 famílias. Poaceae (45 espécies), Cyperaceae (28) e Asteraceae (15) perfazem $50 \%$ do total de espécies e se destacaram como as famílias mais ricas. A riqueza de espécies ressalta a diversidade da vegetação de várzea. Isso, associado à fragilidade e à acelerada conversão dessas áreas naturais, salienta a importância e a urgência de conservação dessa tipologia.

Palavras-chave: Florística; várzea; planície de inundação; rio Iguaçu.
\end{abstract}

\begin{abstract}
Floristic composition of the a floodplain in Balsa Nova county, Paraná State, Brazil. The objective of this research was to study the floristic composition on a Iguaçu river floodplain with area of 4,4 hectares, located at Balsa Nova municipality, Parana state, Brazil, and also improve improving the collections of herbaria. For the floristic surveys, observations were done every 15 days by collecting material for herborization and botanical identification. The checklist included 176 species, 103 genera and 46 families, with Poaceae (45), Cyperaceae (28) and Asteraceae (15), comprising 50\% of all species, were the richest families. The species diversity increases the biological diversity of the floodplains. The fragility of these natural environments associated to the accelerated conversion of wide natural areas, shows the urgency of establishing measures that focus on the conservation and preservation of this natural typology.

Keywords: Floristic; floodplain; Iguaçu river.
\end{abstract}

\section{INTRODUÇÃO}

A Formação Pioneira com Influência Fluvial, também conhecida como vegetação de várzeas, representa uma tipologia relacionada a ambientes naturais de grande fragilidade. Ocorrem em meios com saturação hídrica sazonal a permanente e fisionomicamente sugerem ser muito homogêneas. No entanto, possuem peculiaridades, principalmente com relação à florística, que podem estar relacionadas às diferentes unidades geopedológicas nas quais as espécies ocorrem. Aspectos do meio físico, como o regime hídrico, o tipo de solo e a forma de relevo, juntamente com as características climáticas, podem ter relação direta com a ocorrência e distribuição das espécies na paisagem.

Atualmente, estão ameaçadas pela rápida expansão das atividades agrossilvopastoris, principalmente no Sul do Brasil. Áreas de planícies de inundação, que há pouco tempo encontravam-se recobertas por espécies vegetais nativas, hoje se encontram profundamente modificadas, através do uso com sistemas produtivos. No Paraná, muito pouco se conhece sobre as espécies vegetais desses ambientes e sobre as suas relações com os fatores bióticos e abióticos, um dos motivos pelos quais se realizou o presente trabalho. 


\section{OBJETIVOS}

Foram objetivos deste estudo, conhecer a flora da Formação Pioneira com Influência Fluvial (várzea) da planície de inundação do rio Iguaçu na localidade Ponte dos Arcos, Balsa Nova, Paraná, e incrementar coleções de herbários com material botânico dessa vegetação.

\section{REVISÃO BIBLIOGRÁFICA}

As Formações Pioneiras com Influência Fluvial (IBGE, 1992) também são conhecidas como campos edáficos (KLEIN; HATSCHBACH, 1962), campos de inundação (KLEIN, 1964), brejos (JOLY, 1970) e várzeas (MAACK, 1981). Correspondem às comunidades vegetais que se desenvolvem especialmente sobre planícies aluviais ou margens de lagoas (LEITE; KLEIN, 1990). Podem, ainda, segundo Rodrigues (2000), ser denominadas de Formação Ribeirinha com Influência Fluvial Permanente ou Sazonal, segundo proposta de classificação relacionada aos tipos de vegetação de ambientes ciliares, também conhecidos como ribeirinhos.

As enchentes e/ou o encharcamento do solo, nesses ambientes, são elementos naturais de especificidade ambiental e contribuem para a heterogeneidade vegetacional das áreas onde estão situadas, em função das diferentes frequências, intensidades e durações com que ocorrem, e da quantidade e qualidade dos sedimentos que são carreados. Dessa forma, a condição climácica dessas áreas é caracterizada por um grande número de espécies e de indivíduos dos grupos ecológicos mais iniciais da sucessão, como resultado da ocorrência histórica e repetida desses eventos naturais de perturbações (RODRIGUES, 2000).

Em face da deposição de materiais durante as cheias dos rios, as várzeas geralmente estão sobre terrenos férteis, porém com áreas periódica ou permanentemente inundadas (LEITE; KLEIN, 1990). A água em excesso, nesses ambientes, constitui-se num elemento inibidor e seletivo da vegetação, impedindo o desenvolvimento de uma cobertura vegetal mais exuberante e heterogênea (LEITE; KLEIN, 1990). O solo inundado, durante todo o ano ou durante alguns meses, impede o acesso de ar, necessário à respiração das raízes, criando um ambiente anaeróbico ou com baixa oxigenação, no qual somente certas espécies, selecionadas pelas adaptações que possuem, conseguem sobreviver (JOLY, 1970; CAETANO, 2003).

Em face dessas peculiaridades, o ambiente das várzeas geralmente apresenta grande riqueza biológica, com propriedades de ambos os ecossistemas, o aquático e o terrestre (PITELLI, 1986). São hábitats para pequenos peixes, aves e outras formas de vida e apresentam funções hidrológicas relacionadas à amenização de picos de inundação e de recarga do solo (NATIONAL RESEARCH COUNCIL, 1992). As espécies vegetais que as constituem são elementos importantes para a manutenção do equilíbrio ecológico, atuando na proteção contra a erosão bem como para a conservação da fauna desses ambientes (IRGANG; GASTAL JÚNIOR, 1996).

Nessas áreas podem ser encontrados vegetais que suportam desde submergências ocasionais até o hábito exclusivamente aquático (POMPEU; MOSCHINI-CARLOS, 2003). Entre as espécies de plantas herbáceas que crescem nas áreas inundáveis, são comuns diferentes estratégias para sobrevivência e reprodução no meio saturado. Entre essas ações estão a resistência de sementes e esporos contra inundações e secas e os ciclos reprodutivos curtos, com altas taxas de reprodução, essenciais para a colonização dos hábitats efêmeros da planície de inundação; a propagação vegetativa através de fragmentação e o crescimento a partir de estolões; a elevada produtividade, para compensar perdas anuais no meio instável; a tolerância à inundação e à seca, através de variadas adaptações, como formação de aerênquima em raiz e caule, pneumatóforos e o inchamento de caules submersos para flutuações; e a plasticidade morfológica e fisiológica das espécies em fases úmidas e secas, principalmente relacionadas com a forma (heterofilia) e o tamanho da área foliar e com a quantidade de aerênquima produzida (JUNK, 1980; JUNK; PIEDADE, 1993; JUNK; PIEDADE, 1997).

A vegetação de várzeas é constituída principalmente por espécies de Poaceae (gramíneas) e Cyperaceae que, em conjunto, apresentam-se com grande uniformidade fitofisionômica (KLEIN; HATSCHBACH, 1962). Destacam-se também espécies de Lentibulariaceae, Droseraceae, Lycopodiaceae, Mayacaceae, Eriocaulaceae e Juncaceae (KLEIN, 1964; MAACK, 1981). 
$\mathrm{Na}$ literatura técnico-científica, pouco se conhece sobre essas fisionomias e as espécies constituintes desses ambientes. As informações disponíveis quase sempre estão relacionadas a caracterizações genéricas, com citação apenas das espécies que ocorrem com maior frequência. Informações mais específicas sobre a florística e a estrutura da vegetação, bem como da relação com os diferentes tipos de solos, até o momento são pouco conhecidas. Vale ressaltar que, em sua totalidade, as relações obtidas com os solos se fazem em elevados níveis de abstração, praticamente desconsiderando os atributos dos solos e sua inserção no modelado da paisagem, o que, na maioria das vezes, fragiliza a informação.

Dentre os trabalhos realizados no Brasil, com enfoque em planícies de inundações representadas principalmente por comunidades herbáceo-arbustivas, citam-se o de Braga (1962), Klein; Hatschbach (1970/1971), Imaguire (1985), Bueno et al. (1987), Junk; Piedade (1993), Silva et al. (1997), Campos; Souza (1997), Souza et al. (1997) e Rosa; Irgang (1998). Além desses trabalhos, Cervi et al. (1983), Irgang et al. (1984), Pedralli et al. (1985), Oliveira et al. (1988), Pedralli et al. (1993), Irgang; Gastal Júnior (1996), Gastal Júnior; Irgang (1997), Thomaz et al. (1999) e Maltchik et al. (2002) realizaram levantamentos específicos da vegetação macrófita aquática, listando, entre outras, espécies com ocorrência em vegetação de várzeas do Sul do Brasil.

As planícies de inundação e a vegetação sobre elas estabelecida, apesar de consideradas como áreas de preservação permanente (APP) pela legislação ambiental brasileira (BRASIL, 1965; 2001; 2002), estão ameaçadas pela conversão de suas áreas, principalmente, para atividades agrossilvopastoris. Em função disso, alterações que parecem irreversíveis, em nível de espécie, comunidade ou do ecossistema como um todo, estão ocorrendo nesses frágeis ambientes (JUNK, 1993). Essas alterações, que podem ser de natureza biológica (como a remoção da vegetação natural e introdução de plantas não nativas e/ou invasoras), química (através da descarga de elementos químicos) ou física (resultando na eliminação de feições da topografia e da hidrologia do ecossistema para a prática de sistemas de produção, drenagem e construção de barragens, entre outras obras), frequentemente ocorrem juntas e podem resultar em impactos cumulativos (PITELLI, 1986; NATIONAL RESEARCH COUNCIL, 1992).

Recentemente, o Instituto Ambiental do Paraná (IAP), a Secretaria de Estado do Meio Ambiente e Recursos Hídricos (SEMA) e o Instituto Brasileiro do Meio Ambiente e dos Recursos Naturais Renováveis (IBAMA), preocupados com os níveis crescentes de degradação das várzeas e dos ambientes interdependentes, bem como com as consequências advindas desse processo, com implicações relacionadas principalmente com a disponibilidade futura de água, mobilizaram-se para a criação de uma lei que pudesse salvaguardar as áreas úmidas. Essa lei se concretizou na forma de uma resolução (IBAMA/SEMA/IAP, 2008) para o estado do Paraná. Entre outras providências, determina que, nas áreas úmidas em que foram realizadas intervenções de natureza antrópica, os empreendedores ou proprietários deverão adotar práticas ambientalmente sustentáveis, como eliminar o uso de agrotóxicos, suspender a abertura de canais de drenagem, restringir a adubação, retirar os animais domésticos da área e recuperar imediatamente as áreas mineradas. Com a implantação dessa legislação, espera-se que os órgãos ambientais possam orientar ou coibir o uso de áreas de várzeas anteriormente modificadas, bem como preservar integralmente os últimos remanescentes naturais dessa tipologia no estado.

\section{MATERIAL E MÉTODOS}

\section{Caracterização da área de estudo}

A área de estudo está localizada no município de Balsa Nova, Paraná $\left(25^{\circ} 33,29^{\prime} \mathrm{S}, 49^{\circ} 48,34^{\prime} \mathrm{W}\right)$ e é conhecida como Ponte dos Arcos, denominação devida à presença de um viaduto transpondo a porção final do rio dos Papagaios, em segmento anterior à sua foz no rio Iguaçu. Ela se encontra na Área de Proteção Ambiental da Escarpa Devoniana.

Relacionada ao histórico de formação dos dois rios, encontra-se uma planície de inundação coberta por vegetação de várzea (Formação Pioneira com Influência Fluvial). A planície, entre outras funções, atua como receptora das águas de transbordamento dos rios nas grandes cheias e dos fluxos hídricos provenientes de vertentes, situadas no entorno e cobertas por estepes gramíneo-lenhosas (campos naturais). Dessa forma, atuam favorecendo a regulação hidrológica fluvial. Ainda com relação à planície, está limitada por florestas ciliares dos rios Iguaçu e dos Papagaios, por cultivos agrícolas, pastagens e 
mineração de areia. Essas atividades, juntamente com plantios de Pinus sp. nas imediações, constituem os principais fatores de antropismo da localidade.

Dados do SIMEPAR entre 1997 e 2008, da estação 25474946 na Lapa, município pertencente à mesma bacia hidrográfica e em condições fisiográficas, litológicas e altitudinais semelhantes às da área deste estudo, registraram média anual de umidade relativa do ar igual a $86,7 \%$. A temperatura média anual foi de $17,6{ }^{\circ} \mathrm{C}$, a temperatura média das máximas de $19,3{ }^{\circ} \mathrm{C}$ e a mínima de $15,8{ }^{\circ} \mathrm{C}$. Do valor médio total de precipitação $(1410 \mathrm{~mm})$ registrado no intervalo de anos considerado, 61,5\% concentraram-se nos meses de primavera e verão e $38,5 \%$ nos meses de outono e inverno.

Especificamente com relação à área de estudo, está situada sobre arenitos do grupo Itararé, a cerca de 820 m s.n.m, compreendendo aproximadamente 4,4 ha.

\section{Levantamento florístico}

Para o estudo florístico, foram realizadas visitas quinzenais entre abril de 2005 e abril de 2007, com campanhas de campo com duração de um dia, quando foram realizadas caminhadas intensivas. Foram coletadas todas as espécies herbáceas e subarbustivas encontradas férteis e pertencentes às angiospermas e pteridófitas. Os procedimentos adotados para a coleta e herborização seguiram as recomendações básicas para trabalhos dessa natureza, de acordo com o Instituto de Botânica (1989) e o IBGE (1992).

Após a secagem, os materiais foram submetidos à determinação em nível de família, gênero e espécie. Utilizaram-se, para isso, bibliografia específica, comparações com exsicatas de herbários e consultas a especialistas. Os materiais foram registrados e incorporados à coleção do Herbário do Museu Botânico Municipal (MBM). Duplicatas foram depositadas nos herbários da Escola de Floresta (EFC) e do Departamento de Botânica (UPCB), ambos da Universidade Federal do Paraná (UFPR).

O sistema de classificação adotado para as famílias de angiospermas foi o de Cronquist (1988), com exceção de Liliaceae sensu lato, para a qual foram consideradas as famílias Alstroemeriaceae, Amaryllidaceae, Hypoxidaceae e Liliaceae, de acordo com Dahlgren et al. (1985). Para as pteridófitas, utilizaram-se os sistemas de Smith et al. (2006) e de Tryon e Tryon (1982). Esses sistemas são utilizados nos herbários em que os materiais foram depositados e em muitas monografias publicadas, possibilitando, dessa forma, a realização de comparações florísticas.

Para a análise da similaridade, foi utilizado o índice de Jaccard (MUELLER-DOMBOIS; ELLENBERG, 1974), que expressa a relação entre as espécies comuns que ocorrem em duas diferentes áreas. Em análises de dados qualitativos, esse índice se caracteriza como um dos mais empregados (MATTEUCCI; COLMA, 1982). Foram comparados os resultados florísticos deste trabalho com os estudos de Junk; Piedade (1993), Silva et al. (1997) e Rosa; Irgang (1998), que abordaram a vegetação herbáceo-subarbustiva em áreas de planície de inundação localizadas no Brasil.

\section{RESULTADOS E DISCUSSÃO}

Foram registradas 176 espécies, 103 gêneros e 46 famílias na vegetação de várzea da planície de inundação (Tabela 1). Poaceae (45), Cyperaceae (28) e Asteraceae (15) foram as mais ricas (Figura 1). Correspondem a 50\% do total de espécies levantadas nessa tipologia. Em outros trabalhos realizados em planícies de inundação, Poaceae e Cyperaceae também se destacaram como as de maior riqueza (BRAGA, 1962; KLEIN; HATSCHBACH, 1962, 1970/1971; KLEIN, 1979; JUNK; PIEDADE, 1993; SILVA et al., 1997). Por esses resultados, observa-se que estão entre as de maior riqueza específica em áreas de várzeas brasileiras.

Com relação às formas biológicas, na fisionomia e na florística da vegetação de várzea são dominantes as herbáceas (149 sp. - 84,5\%). Também ocorrem subarbustivas (17 sp. - 10\%) e trepadeiras (5 sp. - 3\%), porém distribuídas de forma bastante esparsa. As espécies subarbustivas só foram observadas em agrupamentos maiores nas porções da planície onde há maior deposição de sedimentos, por influência de cones de dejeção, formados a partir da porção média das vertentes convergentes, onde ocorrem os Gleissolos Melânicos. 


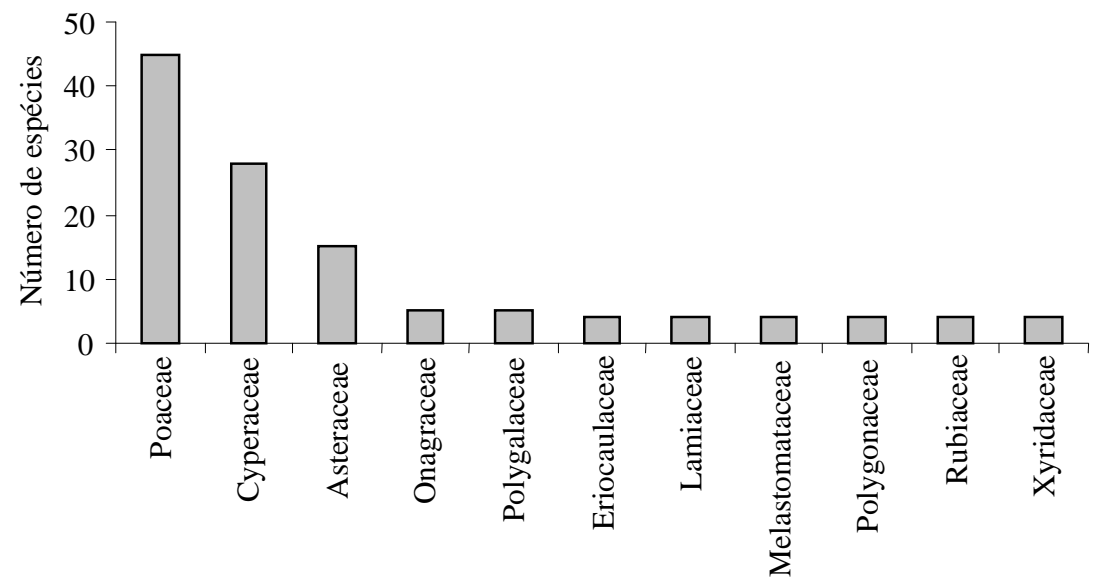

Figura 1. Famílias mais ricas $(70 \%)$ da vegetação de várzea.

Figure 1. The richest families (70\%) of the floodplain vegetation.

A heterogeneidade do meio físico da planície de inundação na área da Ponte dos Arcos é um dos fatores determinantes da riqueza florística evidenciada. Nela existem paleodiques e paleocanais resultantes da dinâmica dos ambientes fluviais dos rios Iguaçu e dos Papagaios, além da porção distal (final) de cones de dejeção formados pela deposição dos sedimentos transportados das partes mais elevadas das vertentes. Sobre e entre essas feições, apesar de pouco aparentes, existem diferenças, como, por exemplo, quanto aos tipos de solos (Organossolos Háplicos e Gleissolos Melânicos), de sedimentos depositados e de saturação de água que podem determinar o estabelecimento das espécies. Junk; Piedade (1993) também verificaram em Manaus (AM) que a riqueza de espécies na planície de inundação do rio Amazonas estava relacionada com a diversidade de hábitats do ambiente de estudo. A essa diversidade relacionaram a existência de ambientes com distinções expressivas nos regimes de saturação hídrica dos diferentes tipos de solos.

Tabela 1. Pteridófitas e angiospermas da Formação Pioneira com Influência Fluvial (várzeas) do rio Iguaçu (Balsa Nova, Paraná, Brasil), com informações sobre o hábitat (te: terrícola), forma de vida (sub: subarbustiva, he: herbácea, tr: trepadeira), número do coletor (C.Kozera) e número de registro no herbário MBM.

Table 1. Pteridophytes and flowering plants of the floodplain Iguaçu river with informations about habitat (te: terrestrial), life form (sub: shrub, he: herb, tr: vine), collector number (C.Kozera) and register number at the Herbarium MBM.

\begin{tabular}{|c|c|c|c|c|c|}
\hline Famílias & Espécies & Hábitat & $\begin{array}{l}\text { Forma } \\
\text { de vida }\end{array}$ & $\begin{array}{l}\mathrm{N}^{\mathbf{0}} \text { do } \\
\text { coletor }\end{array}$ & MBM \\
\hline & PTERIDÓFITAS & & & & \\
\hline Blechnaceae & Blechnum cordatum (Desv.) Hieron. & te & he & 2004 & 319200 \\
\hline \multirow{2}{*}{ Lycopodiaceae } & Lycopodiella alopecuroides (L.) Cranfill & te & he & 2185 & 319201 \\
\hline & Lycopodiella caroliniana (L.) Pic. Serm. & te & he & 2186 & 319202 \\
\hline Osmundaceae & Osmunda regalis $\mathrm{L}$. & te & he & $*$ & $*$ \\
\hline \multirow[t]{2}{*}{ Salviniaceae } & Azolla caroliniana Willd. & te & he & 2434 & 319193 \\
\hline & Salvinia auriculata Aubl. & te & he & 2433 & 319192 \\
\hline Thelypteridaceae & $\begin{array}{l}\text { Thelypteris rivularioides (Fée) Abbiatti } \\
\text { ANGIOSPERMAS }\end{array}$ & te & he & 2038 & 319195 \\
\hline Acanthaceae & Hygrophila costata Nees & te & sub & 2027 & 313814 \\
\hline Alismataceae & $\begin{array}{l}\text { Echinodorus grandiflorus (Cham. \& Schltdl.) } \\
\text { Micheli }\end{array}$ & te & he & 2572 & 313818 \\
\hline Alstromeriaceae & Alstroemeria apertiflora Baker & te & he & 2659 & 313817 \\
\hline
\end{tabular}

FLORESTA, Curitiba, PR, v. 39, n. 2, p. 309-322, abr./jun. 2009. 


\begin{tabular}{|c|c|c|c|c|c|}
\hline \multirow[t]{3}{*}{ Apiaceae } & Eryngium ebracteatum Lam. & te & he & 2576 & 313800 \\
\hline & Eryngium eburneum Decne. & te & he & 2087 & 313801 \\
\hline & Centella asiatica (L.) Urb. & te & he & 3133 & 317889 \\
\hline Apocynaceae & Mandevilla widgrenii C. Ezcurra & te & sub & 2787 & 318044 \\
\hline Asclepiadaceae & Oxypetalum pachygynum Decne. & te & $\operatorname{tr}$ & 2109 & 313823 \\
\hline \multirow{15}{*}{ Asteraceae } & Aster squamatus (Spreng.) Hieron. & te & he & 2032 & 308109 \\
\hline & $\begin{array}{l}\text { Austroeupatorium rosmarinaceum (Cabrera \& } \\
\text { Vittet) R.M. King \& H. Rob. }\end{array}$ & te & sub & 2105 & 313947 \\
\hline & Baccharis spicata (Lam.) Baill. & te & sub & 2235 & 308112 \\
\hline & Baccharis trimera (Less.) DC. & te & he, sub & 2030 & 315151 \\
\hline & Erigeron tweediei Hook. \& Arn. & te & he & 3052 & 317966 \\
\hline & Gamochaeta filaginea (DC.) Cabrera & te & he & 2498 & 313966 \\
\hline & $\begin{array}{l}\text { Hatschbachiella tweedieana (Hook. \& Arn.) R.M. } \\
\text { King \& H. Rob. }\end{array}$ & te & sub & 3136 & 317931 \\
\hline & Hypochaeris lutea Britton & te & he & 2307 & 313970 \\
\hline & Lessingianthus glabratus (Less.) H. Rob. & te & he, sub & 2994 & 317896 \\
\hline & $\begin{array}{l}\text { Lessingianthus polyphyllus (Sch. Bip. ex Baker) } \\
\text { H. Rob. }\end{array}$ & te & he, sub & 2828 & 317894 \\
\hline & Mikania micrantha Kunth & te & $\operatorname{tr}$ & 1984 & 313974 \\
\hline & Pluchea oblongifolia DC. & te & he & 2888 & 317947 \\
\hline & Solidago chilensis Meyen & te & he & 1991 & 308122 \\
\hline & Vernonanthura chamaedrys (Less.) H. Rob. & te & sub & 2000 & 315162 \\
\hline & Vernonanthura nudiflora (Less.) H. Rob. & te & sub & 2117 & 335477 \\
\hline \multirow[t]{3}{*}{ Begoniaceae } & Begonia cucullata Willd. & te & he & 2698 & 319219 \\
\hline & Begonia fischeri Schrank & te & he & 1990 & 313831 \\
\hline & Begonia setosa Klotzsch & te & he & 2784 & 319218 \\
\hline \multirow[t]{3}{*}{ Campanulaceae } & Lobelia exaltata Pohl & te & he & 2031 & 308123 \\
\hline & Lobelia nummularioides Cham. & te & he & 2101 & 308157 \\
\hline & Pratia hederacea (Cham.) G. Don & te & he & 3006 & 318012 \\
\hline \multirow[t]{24}{*}{ Cyperaceae } & $\begin{array}{l}\text { Ascolepis brasiliensis (Kunth) Benth. ex C.B. } \\
\text { Clarke }\end{array}$ & te & he & 2883 & 319244 \\
\hline & Carex brasiliensis A. St.-Hil. & te & he & 2649 & 319246 \\
\hline & $\begin{array}{l}\text { Carex longii var. meridionalis (Kük.) G.A. } \\
\text { Wheeler }\end{array}$ & te & he & 2515 & 319245 \\
\hline & Cyperus haspan $\mathrm{L}$. & te & he & 2113 & 318679 \\
\hline & Cyperus intricatus Schrad. ex Schult. & te & he & 1982 & 318690 \\
\hline & Cyperus rigens J. Presl \& C. Presl & te & he & 2526 & 318683 \\
\hline & Cyperus virens Michx. & te & he & 2012 & 318692 \\
\hline & Eleocharis flavescens (Poir.) Urb. & te & he & 2014 & 318638 \\
\hline & Eleocharis minima Kunth & te & he & 2798 & 318634 \\
\hline & Eleocharis montana (Kunth) Roem. \& Schult. & te & he & 2800 & 318640 \\
\hline & Eleocharis nudipes (Kunth) Palla & te & he & 2390 & 318636 \\
\hline & Eleocharis cf. sellowiana Kunth & te & he & 2187 & 318639 \\
\hline & Fimbristylis autumnalis (L.) Roem. \& Schult. & te & he & 2948 & 318662 \\
\hline & Fimbristylis complanata (Retz.) Link & te & he & 2837 & 343500 \\
\hline & Lipocarpha humboldtiana Nees & te & he & 2281 & 308108 \\
\hline & Pycreus lanceolatus (Poir.) C.B. Clarke & te & he & 2979 & 318677 \\
\hline & Pycreus niger (Ruiz \& Pav.) Cufod. & te & he & 3047 & 318671 \\
\hline & Rhynchospora asperula (Nees) Steud. & te & he & 2597 & 318646 \\
\hline & Rhynchospora aff. eximia (Nees) Boeck. & te & he & 2971 & 318645 \\
\hline & Rhynchospora corymbosa (L.) Britton & te & he & 3344 & 343498 \\
\hline & Rhynchospora emaciata (Nees) Boeck. & te & he & 2126 & 318661 \\
\hline & Rhynchospora gigantea Link & te & he & 1987 & 318651 \\
\hline & Rhynchospora marisculus Lindl. ex Nees & te & he & 3129 & 318653 \\
\hline & Rhynchospora velutina (Kunth) Boeck. & te & he & 2736 & 318648 \\
\hline
\end{tabular}




\begin{tabular}{|c|c|c|c|c|c|}
\hline & Scirpus validus Vahl & te & he & 2420 & 318630 \\
\hline & Scleria hirtella Sw. & te & he & 2061 & 308105 \\
\hline & Scleria setacea Poir. & te & he & 3128 & 318673 \\
\hline & Scleria verticillata Muhl. ex Willd. & te & he & 2839 & 318676 \\
\hline \multirow[t]{4}{*}{ Eriocaulaceae } & Eriocaulon ligulatum (Vellozo) L.B. Sm. & te & he & 2241 & 308117 \\
\hline & Eriocaulon sellowianum Kunth & te & he & 2814 & 335435 \\
\hline & Paepalanthus catharinae Ruhland & te & he & 2041 & 313938 \\
\hline & Syngonanthus caulescens (Poir.) Ruhland & te & he & 2015 & 313935 \\
\hline Euphorbiaceae & Phyllanthus niruri L. & te & he & 2571 & 313944 \\
\hline Fabaceae & $\begin{array}{l}\text { Vigna peduncularis var. clitorioides (Mart. ex } \\
\text { Benth.) Maréchal, Mascherpa \& Stainier }\end{array}$ & te & $\operatorname{tr}$ & 3020 & 318005 \\
\hline Gentianaceae & Schultesia australis Griseb. & te & he & 2914 & 319221 \\
\hline Haloragaceae & Myriophyllum aquaticum (Vell.) Verdc. & te & he & * & $*$ \\
\hline \multirow[t]{2}{*}{ Hypericaceae } & Hypericum brasiliense Choisy & te & sub & 2174 & 308158 \\
\hline & Hypericum denudatum A. St.-Hil. & te & sub & 2199 & 308135 \\
\hline \multirow[t]{3}{*}{ Iridaceae } & Sisyrinchium hasslerianum Baker & te & he & 2392 & 314017 \\
\hline & Sisyrinchium micranthum Cav. & te & he & 2633 & 314020 \\
\hline & Sisyrinchium sp. & te & he & 2795 & 318027 \\
\hline \multirow[t]{2}{*}{ Juncaceae } & Juncus densiflorus Kunth & te & he & 2723 & 331959 \\
\hline & Juncus microcephalus Kunth & te & he & 2724 & 331960 \\
\hline \multirow[t]{4}{*}{ Lamiaceae } & Hyptis balansae Briq. & te & he & 1992 & 314024 \\
\hline & Hyptis fasciculata Benth. & te & he & 1994 & 314023 \\
\hline & Rhabdocaulon lavanduloides (Benth.) Epling & te & sub & 1996 & 314028 \\
\hline & Scutellaria racemosa Pers & te & he & 2542 & 314031 \\
\hline \multirow[t]{3}{*}{ Lentibulariaceae } & Utricularia praelonga A. St.-Hil. \& Girard & te & he & 2482 & 327452 \\
\hline & Utricularia tricolor A. St.-Hil. & te & he & 2697 & 327442 \\
\hline & Utricularia sp. (flores brancas) & te & he & 2815 & $*$ \\
\hline Linaceae & Linum littorale A. St.-Hil. var. littorale & te & he & 2279 & 335429 \\
\hline Lythraceae & Heimia myrtifolia Cham. \& Schltdl. & te & sub & 2196 & 308141 \\
\hline Malpighiaceae & Janusia guaranitica (A. St.-Hil.) A. Juss. & te & $\operatorname{tr}$ & 2874 & 318034 \\
\hline Mayacaceae & Mayaca sellowiana Kunth & te & he & 2028 & 308126 \\
\hline \multirow[t]{4}{*}{ Melastomataceae } & Rhynchanthera brachyrhyncha Cham. & te & he & 2786 & 319209 \\
\hline & Tibouchina cerastifolia Cogn. & te & he & 3132 & 335454 \\
\hline & Tibouchina gracilis (Bonpl.) Cogn. & te & he & 2534 & 335451 \\
\hline & Tibouchina ursina (Cham.) Cogn. & te & he, sub & 2019 & 308147 \\
\hline Menyanthaceae & Nymphoides indica (L.) Kuntze & te & he & $*$ & $*$ \\
\hline Mimosaceae & Mimosa lanata Benth. & te & sub & 2083 & 313891 \\
\hline \multirow[t]{5}{*}{ Onagraceae } & Ludwigia elegans (Cambess.) H. Hara & te & Sub & 2006 & 313893 \\
\hline & Ludwigia longifolia (DC.) H. Hara & te & sub & 2037 & 313895 \\
\hline & Ludwigia martii (Micheli) Ramamoorthy & te & he & 2872 & 318045 \\
\hline & Ludwigia nervosa (Poir.) H. Hara & te & sub & 2702 & 318047 \\
\hline & Ludwigia sericea (Cambess.) H. Hara & te & sub & 2040 & 308124 \\
\hline \multirow[t]{2}{*}{ Orchidaceae } & Habenaria edwallii Cogn. & te & he & 2440 & 319230 \\
\hline & Habenaria johannensis Barb. Rodr. & te & he & 2915 & 319226 \\
\hline \multirow[t]{10}{*}{ Poaceae } & Andropogon lateralis Nees & te & he & 2578 & 321258 \\
\hline & Andropogon macrothrix Trin. & te & he & 2405 & 321259 \\
\hline & Andropogon virgatus Desv. ex Ham. & te & he & 2892 & 321263 \\
\hline & $\begin{array}{l}\text { Arundinella hispida (Humb. \& Bonpl. ex Willd.) } \\
\text { Kuntze }\end{array}$ & te & he & 1980 & 308101 \\
\hline & Axonopus polystachyus G.A. Black & te & he & 2969 & 319296 \\
\hline & Axonopus siccus (Nees) Kuhlm. & te & he & 2996 & 319293 \\
\hline & Briza calotheca (Trin.) Hack. & te & he & 2421 & 325390 \\
\hline & Calamagrostis viridiflavescens (Poir.) Steud. & te & he & 2194 & 308107 \\
\hline & Coelorachis aurita (Steud.) A. Camus & te & he & 2193 & 343502 \\
\hline & Danthonia secundiflora J. Presl & te & he & 2385 & 319251 \\
\hline
\end{tabular}

FLORESTA, Curitiba, PR, v. 39, n. 2, p. 309-322, abr./jun. 2009. 


\begin{tabular}{|c|c|c|c|c|c|}
\hline & $\begin{array}{l}\text { Dichanthelium sabulorum (Lam.) Gould \& C.A. } \\
\text { Clark }\end{array}$ & te & he & 2135 & 321168 \\
\hline & $\begin{array}{l}\text { Dichanthelium surrectum (Chase ex Zuloaga \& } \\
\text { Marrone) Zuloaga }\end{array}$ & te & he & 1989 & 321171 \\
\hline & Eragrostis airoides Nees & te & he & 2946 & 326959 \\
\hline & Eragrostis bahiensis Schrad. ex Schult. & te & he & 2585 & 325398 \\
\hline & Eriochrysis cayennensis P. Beauv. & te & he & 2280 & 308103 \\
\hline & Eriochrysis cf. holcoides (Nees) Kuhlm. & te & he & 2290 & 326961 \\
\hline & Gymnopogon burchellii (Munro ex Döll) Ekman & te & he & 2939 & 325408 \\
\hline & Hemarthria altissima (Poir.) Stapf \& C.E. Hubb. & te & he & 2033 & 343510 \\
\hline & Imperata brasiliensis Trin. & te & he & 2527 & 319253 \\
\hline & Leersia hexandra Sw. & te & he & 2011 & 308096 \\
\hline & Luziola bahiensis (Steud.) Hitchc. & te & he & 2711 & 325406 \\
\hline & Otachyrium versicolor (Döll) Henrard & te & he & 2267 & 308097 \\
\hline & Panicum glabripes Döll & te & he & 2598 & 321159 \\
\hline & Panicum parvifolium Lam. & te & he & 2119 & 321151 \\
\hline & Panicum prionitis Nees & te & he & 2808 & 321152 \\
\hline & Panicum pseudisachne $\mathrm{Mez}$ & te & he & 2049 & 321170 \\
\hline & Panicum schwackeanum $\mathrm{Mez}$ & te & he & 2965 & 321181 \\
\hline & Paspalum cordatum Hack. & te & he & 2825 & 326955 \\
\hline & Paspalum ellipticum Döll & te & he & 2590 & 319992 \\
\hline & Paspalum erianthoides Lindm. & te & he & 2313 & 319991 \\
\hline & Paspalum exaltatum J. Presl & te & he & 2899 & 319979 \\
\hline & Paspalum falcatum Nees ex Steud. & te & he & 2740 & 319975 \\
\hline & Paspalum flaccidum Nees & te & he & 2647 & 319982 \\
\hline & $\begin{array}{l}\text { Paspalum intermedium Munro ex Morong \& } \\
\text { Britton }\end{array}$ & te & he & 2587 & 319990 \\
\hline & Paspalum maculosum Trin. & te & he & 3040 & 319301 \\
\hline & Paspalum plicatulum Michx. & te & he & 2251 & 319972 \\
\hline & Paspalum polyphyllum Nees ex Trin. & te & he & 2252 & 319968 \\
\hline & Saccharum aff. angustifolium (Nees) Trin. & te & he & 2908 & 325396 \\
\hline & Saccharum asperum (Nees) Steud. & te & he & 3378 & 325392 \\
\hline & Saccharum villosum Steud. & te & he & 2530 & 325393 \\
\hline & Sacciolepis vilvoides (Trin.) Chase & te & he & 2900 & 321157 \\
\hline & Schizachyrium condensatum (Kunth) Nees & te & he & 2084 & 321257 \\
\hline & Setaria parviflora (Poir.) Kerguélen & te & he & 2618 & 325391 \\
\hline & Steinchisma decipiens (Nees ex Trin.) W.V. Br & te & he & 2586 & 321156 \\
\hline & Trachypogon montufarii (Kunth) Nees & te & he & 3026 & 326952 \\
\hline Polygalaceae & Monnina tristaniana A. St.-Hil. & te & he & 2263 & 313870 \\
\hline & Polygala brasiliensis $\mathrm{L}$. & te & he & 3210 & 318023 \\
\hline & Polygala longicaulis Kunth & te & he & 2489 & 313872 \\
\hline & Polygala molluginifolia A. St.-Hil. \& Moq. & te & he & 2541 & 313874 \\
\hline & Polygala moquiniana A. St.-Hil. \& Moq. & te & he & 2490 & 313873 \\
\hline Polygonaceae & Polygonum hydropiperoides Michx. & te & he & 2247 & 308154 \\
\hline & Polygonum meisnerianum Cham. \& Schltdl. & te & he & 2580 & 319214 \\
\hline & Polygonum punctatum Elliott & te & he & 2779 & 319213 \\
\hline & Polygonum rubricaule Cham. & te & he & 2007 & 308156 \\
\hline Pontederiaceae & Heteranthera zosterifolia Mart. & te & he & 2275 & 308133 \\
\hline & Pontederia cordata L. & te & he & 2010 & 308155 \\
\hline Potamogetonaceae & Potamogeton sp. & te & he & $*$ & $*$ \\
\hline Rubiaceae & Diodia alata Nees \& Mart. & te & he & 2001 & 313880 \\
\hline & $\begin{array}{l}\text { Oldenlandia salzmannii (DC.) Benth. \& Hook. f. } \\
\text { ex A.B. Jacks. }\end{array}$ & te & he & 2102 & 313883 \\
\hline & Galium equisetoides (Cham. \& Schltdl.) Standl. & te & $\operatorname{tr}$ & 2200 & 308125 \\
\hline & Galium nigroramosum (Ehrend.) Dempster & te & he & 3267 & 335414 \\
\hline
\end{tabular}




\begin{tabular}{llllll}
\hline Scrophulariaceae & Buchnera integrifolia Larrañaga & te & he & 2583 & 313904 \\
& Melasma rhinanthoides (Cham. \& Schltdl.) Benth. & te & he, sub & 2076 & 308128 \\
Solanaceae & Solanum aculeatissimum Jacq. & te & sub & 1998 & 313912 \\
Valerianaceae & Valeriana muelleri Graebn. & te & he & 3299 & 335419 \\
Verbenaceae & Glandularia tweediana Niven & te & he & 2201 & 313916 \\
& Lippia turnerifolia Cham. & te & he & 2435 & 313919 \\
Violaceae & Viola gracillima A. St.-Hill. & te & he & 3336 & 335420 \\
Xyridaceae & Xyris asperula Mart. & te & he & 2674 & 313806 \\
& Xyris jupicai Rich. & te & he & 2924 & 317968 \\
& Xyris schizachne Mart. & te & he & 2735 & 317973 \\
Indeterminada & Xyris tortula Mart. & Te & he & 2566 & 313808 \\
& Indeterminada 1 (herbácea/várzea folhas finas) & Te & he & 3142 & 335455 \\
\hline
\end{tabular}

*: sem coleta de material fértil e/ou sem número de registro no herbário MBM.

$\mathrm{Na}$ Ponte dos Arcos, a heterogeneidade do meio pode ser visualmente percebida pela alteração da fisionomia da vegetação, que é determinada pelo conjunto das formas biológicas, altura e coloração das espécies dominantes e co-dominantes. Esses diferentes grupos de espécies, que definem um mosaico de cores e de formas na paisagem, representam comunidades vegetais distintas, estabelecidas sobre condições diferenciadas do meio, principalmente quanto às características das classes de solos e ao seu grau de saturação hídrica. Rosa; Irgang (1998), estudando a planície de inundação do rio dos Sinos no Rio Grande do Sul (RS), também reconheceram diferentes comunidades vegetais. Segundo esses autores, as comunidades evidenciadas distribuíam-se como manchas na paisagem, refletindo diferentes seres sucessionais e/ou situações diferenciadas, principalmente quanto ao conteúdo de água no solo. Para Junk (1980), a inexistência de uma estrutura uniforme da vegetação na planície é consequência das constantes mudanças das condições ecológicas, decorrentes, principalmente, das flutuações do nível de água.

Em partes mais rebaixadas da planície da Ponte dos Arcos, correspondentes aos paleocanais e onde predominam Organossolos Háplicos, foram observadas duas fisionomias, uma alta e outra baixa. Nas porções da área representadas pela fitofisionomia mais alta (1 a $2 \mathrm{~m})$, ocorrem comunidades geralmente dominadas por uma ou mais das seguintes espécies: Paspalum exaltatum J. Presl, Paspalum falcatum Nees ex Steud, Andropogon lateralis Nees, Andropogon virgatus Desv. ex Ham., Saccharum asperum (Nees) Steud., Saccharum aff. angustifolium (Nees) Trin., Arundinella hispida (Humb. \& Bonpl. ex Willd.) Kuntze, Otachyrium versicolor (Döll) Henrard, Axonopus polystachyus G.A. Black, Eriochrysis cayennensis P. Beauv. (Poaceae), Eryngium ebracteatum Lam. (Apiaceae) e Rhynchospora corymbosa (L.) Britton (Cyperaceae). Da mesma forma como descrito por Klein (1964) e Leite; Klein (1990), as espécies dominantes crescem formando emaranhados de touceiras altas junto a outras espécies, porém menos conspícuas.

Quanto à fitofisionomia mais baixa, foi verificada na porção central da planície, em área relacionada com o principal paleocanal do rio Iguaçu. Nessa porção também ocorrem Organossolos Háplicos, mas permanentemente saturados. A vegetação possui até $1 \mathrm{~m}$ de altura e as espécies herbáceas são dominantes, encontrando-se eventualmente cobertas pelo nível da água na época das grandes cheias. Em determinadas porções desse paleocanal predominam plantas de Eriocaulon ligulatum (Eriocaulaceae), uma espécie com folhas rosuladas, muito conspícua pela abundância em que ocorre. Junto a ela podem ainda ser observadas outras espécies dominantes, como Eleocharis nudipes (Cyperaceae), cujas inflorescências assemelham-se muito à das espécies de Eriocaulaceae, Lipocarpha humboldtiana, espécies diversas de Cyperus (Cyperaceae) e de Xyris (Xyridaceae), Austroeupatorium rosmarinaceum (Asteraceae) e Melasma rhinanthoides (Scrophulariaceae). Em outras porções, separadas da primeira por um cone de dejeção, a fitofisionomia também é dominantemente herbácea, mas caracterizada pela presença de Rhynchospora corymbosa (Cyperaceae), uma espécie graminifólia. Interessante destacar a ocorrência nesses ambientes de Mandevilla widgrenii, uma Apocynaceae com flores róseas que só é percebida na vegetação no período de sua floração. No restante do ano permanece latente, através de órgãos subterrâneos que acumulam reservas, comuns em espécies geófitas (MUELLER-DOMBOIS; ELLENBERG, 1974).

Nas partes mais elevadas da planície, que correspondem aos paleodiques e às porções distais de cones de dejeção, onde ocorrem os Gleissolos Melânicos e há maior variação de flutuação do lençol freático, além de espécies herbáceas também ocorrem subarbustivas, encontrando-se, dentre essas, 
principalmente representantes de Onagraceae (Ludwigia sp.), Asteraceae (Vernonanthura chamaedrys (Less.) H. Rob., Vernonanthura nudiflora (Less.) H. Rob.), Lamiaceae (Hyptis fasciculata Benth., Hyptis balansae Briq., Rhabdocaulon lavanduloides (Benth.) Epling), Hypericaceae e Mimosaceae (Mimosa lanata Benth.). Junto a elas, também ocorrem as espécies anteriormente mencionadas, além de outras de diferentes famílias igualmente comuns na várzea, mas que não são notadas num primeiro instante por ocorrerem nas camadas mais baixas da vegetação, sob a cobertura das mais altas. Entre as espécies dos estratos mais baixos da vegetação, encontram-se Lobelia nummularioides Cham. (Campanulaceae), Syngonanthus caulescens (Poir.) Ruhland (Eriocaulaceae), Utricularia tricolor A. St.-Hil. (Lentibulariaceae), Leersia hexandra Sw., Luziola bahiensis (Steud.) Hitch. (Poaceae), Mayaca sellowiana Kunth (Mayacaceae), Schultesia australis Griseb. (Gentianaceae), Polygala brasiliensis L. (Polygalaceae), Viola gracillima A. St.-Hil. (Violaceae), Galium equisetoides (Cham. \& Schltdl.) Standl. e Oldenlandia salzmannii (DC.) Benth. \& Hook. f. ex A.B. Jacks. (Rubiaceae).

Além dessas, também foram observadas diferentes comunidades vegetais em pequenas bacias de inundação. Entre as espécies presentes nesses ambientes, são comuns as aquáticas, geralmente citadas em listagens florísticas como macrófitas, entre as quais, Azolla caroliniana Willd. e Salvinia auriculata Aubl. (Salviniaceae), Echinodorus grandiflorus (Cham. \& Schltdl.) Micheli (Alismataceae), Eleocharis cf. sellowiana Kunth (Cyperaceae), Myriophyllum aquaticum (Vell.) Verdc. (Haloragaceae), Juncus microcephalus Kunth (Juncaceae), Nymphoides indica (L.) Kuntze (Menyanthaceae), Polygonum hydropiperoides Michx. (Polygonaceae) e Pontederia cordata L. (Pontederiaceae) (CERVI et al., 1983; IRGANG et al., 1984; PEDRALLI et al., 1985; IRGANG; GASTAL JÚNIOR, 1996; THOMAZ et al., 1999; MALTCHIK et al., 2002). Essas espécies são comumente listadas nas fases sucessionais de evolução dos ambientes aquáticos denominadas de hidrossere (IMAGUIRE, 1985), encontrando-se, algumas, na vegetação de várzea da planície de inundação, formação vegetal pioneira e de primeira ocupação, que está em constante sucessão por se encontrar em terrenos com solo em contínuo rejuvenescimento (IBGE, 1992).

As variações na abundância e frequência das espécies dominantes e características das várzeas, conforme mencionado, também foram notadas por Klein; Hatschbach (1970/1971). Segundo esses autores, alterações nas condições edáficas dos ambientes seriam suficientes para influenciar na determinação da ocorrência das espécies mais seletivas.

Ainda com relação à vegetação de várzea estudada, além das comunidades relacionadas com o meio natural, outras duas foram observadas na planície originadas por efeito de atividades antrópicas. A abertura de drenos para uso de parte da planície para a pastagem e a construção de uma estrada para circulação de veículos de mineração de areia, em área de vertentes adjacentes, modificaram as condições hídricas, a saturação iônica e a estrutura do solo, consequentemente, a flora e a vegetação estabelecidas nesses locais. A modificação gradual da florística desses ambientes foi acompanhada a partir do segundo ano dos trabalhos de campo relacionados a este estudo.

Especificamente na área drenada, que corresponde a cerca de $10 \%$ da planície, a fisionomia da vegetação é diferente da várzea como um todo. Não ocorrem subarbustivas e são dominantes poucas espécies, entre as quais, Rhynchospora emaciata (Nees) Boeck., Scleria hirtella Sw. (Cyperaceae) e Andropogon virgatus Desv. ex Ham. (Poaceae). Ao longo da margem do dreno também foi identificada a presença de Centella asiatica (L.) Urb. (Apiaceae), uma espécie herbácea exótica. Conforme observado por Imaguire (1985), em áreas de várzeas modificadas para atividades de interesse econômico, ocorrem modificações florísticas que, em parte, estão relacionadas com o tipo e a intensidade da exploração, sendo o terreno gradualmente ocupado por espécies da vegetação mais próxima, algumas, inclusive, exóticas e/ou invasoras.

Com relação à estrada, a sua construção na porção superior das vertentes intensificou processos erosivos, levando à formação de voçorocas e à deposição de sedimentos em uma pequena área da planície, soterrando a vegetação presente nesse local. O novo ambiente foi colonizado por espécies diferentes daquelas que ocorriam nas condições naturais de saturação hídrica. Anteriormente, eram dominantes espécies de Cyperaceae, principalmente Rhynchospora corymbosa (L.) Britton, substituída nas novas condições de menor saturação por espécies de Poaceae, entre as quais, Dichantelium surrectum (Chase ex Zuloaga \& Marrone) Zuloaga.

Rosa; Irgang (1998) também evidenciaram diversidade de espécies em levantamento florístico da planície de inundação do rio dos Sinos (RS). Da mesma forma como evidenciado neste trabalho, parâmetros relacionados ao conteúdo de água no solo, como o regime hidrológico caracterizado pelas cheias e vazantes e a profundidade do lençol freático, além dos aspectos sucessionais relacionados à 
perturbação antrópica, foram citados pelos autores como os fatores ambientais mais importantes na determinação da distribuição das comunidades e das espécies na planície estudada.

\section{Comparações florísticas}

A riqueza de espécies da vegetação de várzea da Ponte dos Arcos pode ser considerada alta (176 espécies em 4,4 ha) quando comparada a outros trabalhos realizados no Brasil com a mesma tipologia de vegetação, considerando-se apenas os ambientes herbáceos e/ou arbustivos estudados. Junk; Piedade (1993) registraram cerca de 140 espécies distribuídas em áreas de grande sedimentação, periodicamente inundadas e em depressões úmidas e/ou lagos ao longo de cerca de $100 \mathrm{~km}$ de planícies de inundações do rio Amazonas, perto de Manaus (AM). Silva et al. (1997), em São Mateus do Sul (PR), registraram 90 espécies nas lagoas e campos de inundação ao longo de um trecho do rio Iguaçu. Rosa; Irgang (1998) registraram 43 espécies em comunidades aquáticas e herbáceo-arbustivas terrícolas da planície de inundação do rio dos Sinos (RS).

A apresentação de listas simplificadas e de descrições genéricas do ambiente, com citação apenas das espécies mais conspícuas, impossibilitou um maior número de comparações com alguns outros trabalhos também realizados em áreas de inundações (BRAGA, 1962; KLEIN; HATSCHBACH, 1970/1971; IMAGUIRE, 1985; BUENO et al., 1987; CAMPOS; SOUZA, 1997; SOUZA et al., 1997).

Pouco ainda se conhece sobre essa tipologia de vegetação, fato que se pode perceber pelo pequeno número de trabalhos publicados: Braga (1962), Klein; Hatschbach (1970/1971), Imaguire (1985), Bueno et al. (1987), Junk; Piedade (1993), Silva et al. (1997), Campos; Souza (1997), Souza et al. (1997) e Rosa; Irgang (1998). A dificuldade de se trabalhar em meios saturados com água, a complexidade taxonômica dos grupos de espécies características desses ambientes e a pouca importância que é atribuída à vegetação de várzea, provavelmente estão entre os principais motivos que justificam a escassez desses trabalhos.

Com relação à similaridade, os valores obtidos entre as espécies foram inferiores a $25 \%$, valor mínimo sugerido por Mueller-Dombois; Ellenberg (1974) como referencial para identificar comunidades similares quanto à riqueza. A similaridade foi evidenciada apenas entre as famílias da área estudada e aquelas listadas nos trabalhos anteriormente mencionados (Tabela 2).

A localização geográfica das áreas comparadas, sob diferentes condições climáticas, litológicas e pedológicas, além da heterogeneidade das condições ambientais das planícies estudadas, determinadas principalmente pela dinâmica dos rios relacionados, e as diferentes metodologias empregadas, quanto à periodicidade, formas de vida e ambientes considerados nas amostragens, justificam a ausência de similaridade entre as espécies da Ponte dos Arcos e os demais estudos realizados.

Tabela 2. Índice de similaridade de Jaccard entre a Ponte dos Arcos, Balsa Nova (PR), e outras áreas de vegetação de várzea.

Table 2. Jaccard index of similarity between the Arcos bridge, Balsa Nova (PR), and other areas of the vegetation upper a floodplain vegetation.

\begin{tabular}{lllll}
\hline \multirow{2}{*}{ Referência } & \multirow{2}{*}{ Município } & \multirow{2}{*}{ Estado } & & \multicolumn{2}{c}{ Similaridade } \\
\cline { 5 - 6 } \cline { 4 - 5 } & & & Espécies & Famílias \\
\hline Silva et al. (1997) & São Mateus do Sul & PR & $11,739(27)$ & $54,717(29)$ \\
Rosa; Irgang (1998) & Novo Hamburgo & RS & $5,528(11)$ & $29,412(15)$ \\
Junk; Piedade (1993) & Manaus & AM & $1,993(6)$ & $27,419(17)$ \\
\hline N
\end{tabular}

Números entre parênteses correspondem às espécies ou famílias em comum.

\section{CONCLUSÕES}

- Poaceae, Cyperaceae e Asteraceae são as famílias de maior riqueza específica da vegetação de várzea da planície de inundação da localidade Ponte dos Arcos, Balsa Nova (PR).

- A heterogeneidade do meio físico, principalmente quanto a pequenos gradientes altimétricos, que impõem diferenças no regime hídrico dos solos, é um dos fatores determinantes da riqueza de espécies evidenciada nessa tipologia.

- A riqueza de espécies da vegetação de várzea da Ponte dos Arcos é alta, quando comparada a outros trabalhos realizados com o componente herbáceo-subarbustivo de outras planícies de inundação do Brasil. 


\section{REFERÊNCIAS}

BRAGA, R. Contribuição ao estudo fitogeográfico do Estado do Paraná: Serra dos Dourados. Boletim Paranaense de Geografia, Curitiba, v. 6/7, p. 29-41, 1962.

BRASIL. Lei federal n. 4.771, de 15 de setembro de 1965. Institui o novo Código Florestal. Diário

Oficial da União, Brasília, DF, set. 1965. Disponível em: <http://www.planalto.gov.br/ccivil_03/Leis/L4771.htm>. Acesso em: 22/01/2008.

BRASIL. Medida Provisória n. 2166-67, de 24 de agosto de 2001. Altera os arts. $1^{\circ}$, 4ํㅜ 14,16 e 44, e acresce dispositivos à Lei no 4.771 , de 15 de setembro de 1965, que institui o Código Florestal, bem como altera o art. 10 da Lei n. 9.393, de 19 de dezembro de 1996, que dispõe sobre o Imposto sobre a Propriedade Territorial Rural - ITR, e dá outras providências. Diário Oficial da União, Brasília, DF, ago. 2001. Disponível em: <http://www.planalto.gov.br/ccivil_03/MPV/2166-67.htm>. Acesso em: $15 / 03 / 2008$.

BRASIL. RESOLUÇÃO do CONAMA n. 303, de 20 de março de 2002. Dispõe sobre parâmetros, definições e limites de Áreas de Preservação Permanente. Diário Oficial da União, Brasília, DF, mai. 2002. Disponível em: <http://www.mma.gov.br/port/conama/res/res02/res30302.html>. Acesso em: $22 / 01 / 2008$

BUENO, O. L.; NEVES, M. T. M. B. das; OLIVEIRA, M. de L. A. A. de; RAMOS, R. L. D.; STREHL, T. Florística em áreas da margem direita do baixo Jacuí, RS, Brasil. Acta Botanica Brasilica, Porto Alegre, v. 1, n. 2, p. 101-121, 1987.

CAETANO, V. L. Dinâmica sazonal e fitossociologia da vegetação herbácea de uma baixada úmida entre dunas, Palmares do Sul, Rio Grande do Sul, Brasil. Iheringia. Série Botânica, Porto Alegre, v. 58, n. 1, p. 81-102, 2003.

CAMPOS, J. B.; SOUZA, M. C. Vegetação. In: VAZZOLER, A. E. A. M.; AGOSTINHO, A. A.; HAHN, N. S. (Eds.). A planície de inundação do alto rio Paraná: aspectos físicos, biológicos e socioeconômicos. Maringá: EDUEM, 1997. p. 331-342.

CERVI, A. C.; HATSCHBACH, G. G.; GUIMARÃES, O. A. Nota prévia sobre plantas aquáticas (fanerogâmicas) do Estado do Paraná (BR). Boletim do Museu Botânico Municipal, Curitiba, v. 58, p. 1-17, 1983.

CRONQUIST, A. The evolution and classification of flowering plants. New York: New York Botanical Garden, 1988.

DAHLGREN, R. M. T.; CLIFFORD, H. T.; YEO, P. F. The families of the monocotyledons: structure, evolution and taxonomy. Berlin: Springer-Verlag, 1985. 520 p.

GASTAL JUNIOR, C. V. S.; IRGANG, B. E. Levantamento de macrófitas aquáticas do vale do rio Pardo, Rio Grande do Sul, Brasil. Iheringia. Série Botânica, Porto Alegre, v. 49, p. 3-9, 1997.

IMAGUIRE, N. Contribuição ao estudo florístico e ecológico da Fazenda Experimental do Setor de Ciências Agrárias da Universidade Federal do Paraná: 4. Constituição das formações em comunidades estágio e suas evoluções. Revista do Setor de Ciências Agrárias, v. 7, n. 1/2, p. 11-26, 1985.

INSTITUTO BRASILEIRO DE GEOGRAFIA E ESTATÍSTICA (IBGE). Manual técnico da vegetação brasileira. Rio de Janeiro: IBGE, 1992.

INSTITUTO BRASILEIRO DO MEIO AMBIENTE E DOS RECURSOS NATURAIS RENOVÁVEIS; SECRETARIA DE ESTADO DO MEIO AMBIENTE E RECURSOS HÍDRICOS; INSTITUTO AMBIENTAL DO PARANÁ (IBAMA/SEMA/IAP). Resolução conjunta IBAMA/SEMA/IAP n. 5, de 28 de março de 2008. Define critérios para avaliação das áreas úmidas e seus entornos protetivos, normatiza sua conservação e estabelece condicionantes para o licenciamento das atividades neles permissíveis no Estado do Paraná e revoga a Resolução Conjunta IBAMA/SEMA/IAP n. 45, de 25 de setembro de 2007. Diário Oficial do Estado do Paraná, Curitiba, PR, março de 2008. 
INSTITUTO DE BOTÂNICA (São Paulo). Técnicas de coleta, preservação e herborização de material botânico. FIDALGO, O.; BONONI, V. L. R. (Coord.). São Paulo: Instituto de Botânica, 1989. 62 p. (Série Documentos).

IRGANG, B. E.; GASTAL JUNIOR, C. V. de S. Macrófitas aquáticas da planície costeira do Rio Grande do Sul. Porto Alegre, 1996. 290 p.

IRGANG, B. E.; PEDRALLI, G.; WAECHTER, J. L. Macrófitos aquáticos da Estação Ecológica do Taim, Rio Grande do Sul, Brasil. Roessléria, Porto Alegre, v. 6, n. 1, p. 395-404, 1984.

JOLY, A. B. Conheça a vegetação brasileira. São Paulo: Editora da Universidade de São Paulo: Polígono, 1970. $165 \mathrm{p}$.

JUNK, W. J. Áreas inundáveis: um desafio para limnologia. Acta Amazonica, Manaus, v. 10, n. 4, p. 775-795, 1980.

JUNK, W. J. Wetlands of tropical South America. In: WHIGHAM, D.; DYKYJOVÁ, D.; HEJNÝ, S. (Ed.). Wetlands of the world: inventory, ecology and management. London: Kluwer Academic, 1993. v. 1, p. 679-739.

JUNK, W. J.; PIEDADE, M. T. F. Herbaceous plants of the amazon floodplain near Manaus: species diversity and adaptations to the flood pulse. Amazoniana: liminologia et oecologia regionalis systemae fluminis Amazonas, Manaus, v. 12, n. 3/4, p. 467-484, 1993.

JUNK, W. J.; PIEDADE, M. T. F. Plant life in the floodplain with special reference to herbaceous plants. In: JUNK, W. J. (Ed.). The central amazon floodplain: ecology of a pulsing system. Berlim: SpringerVerlag, 1997. p. 147-185. (Ecological Studies, 126).

KLEIN, R. M. Aspectos predominantes da vegetação sul-brasileira. In: CONGRESSO NACIONAL DA SOCIEDADE BOTÂNICA DO BRASIL, 15., 1964, Porto Alegre. Anais... Porto Alegre, 1964. p. 255276.

KLEIN, R. M. Ecologia da flora e vegetação do vale do Itajaí. Sellowia, Itajaí, v. 31, p. 09-164, 1979.

KLEIN, R. M.; HATSCHBACH, G. G. Fitofisionomia e notas sobre a vegetação para acompanhar a planta fitogeográfica do município de Curitiba e arredores (Paraná). Boletim da Universidade do Paraná. Geografia Física, Curitiba, v. 4, p. 1-29, 1962.

KLEIN, R. M.; HATSCHBACH, G. G. Fitofisionomia e notas complementares sobre o mapa fitogeográfico de Quero-Quero (Paraná). Boletim Paranaense de Geociências, Curitiba, v. 28/29, p. 159-188, 1970/1971.

LEITE, P. F.; KLEIN, R. M. Vegetação. In: Geografia do Brasil: região sul. Rio de Janeiro: IBGE, 1990. v. 2, p. 113-150.

MAACK, R. Geografia física do Estado do Paraná. 2. ed. Rio de Janeiro: José Olympio; Curitiba: Secretaria da Cultura e do Esporte do Governo do Estado do Paraná, 1981. 450 p.

MALTCHIK, L.; ROLON, A. S.; GROTH, C. Diversidade de macrófitas aquáticas em áreas úmidas da bacia do rio dos Sinos, Rio Grande do Sul. Pesquisas, São Leopoldo, v. 52, p. 143-154, 2002.

MATTEUCCI, S. D.; COLMA, A. Metodologia para el estudio de la vegetatión. Washington: Secretaria General de la Organización de los Estados Americanos, 1982. 168 p.

MUELLER-DOMBOIS, D.; ELLENBERG, H. Aims and methods of vegetation ecology. New York: J. Wiley and Sons, 1974.

NATIONAL RESEARCH CONCIL. Committee on Restoration of Aquatic Ecosystems - Science, Technology, And Public Policy. Restoration of aquatic systems: science, technology, and public policy. USA: National Academy of Sciences, 1992. $552 \mathrm{p}$. 
OLIVEIRA, M. de L. A. A.; NEVES, M. T. M. B.; STREHL, T.; RAMOS, R. L. D.; BUENO, O. L. Vegetação de macrófitos aquáticos das nascentes do rio Gravataí (Banhado Grande e Banhado Chico Lomã), Rio Grande do Sul, Brasil - levantamento preliminar. Iheringia. Série Botânica, Porto Alegre, v. 38, p. 67-80, 1988.

PEDRALLI, G.; IRGANG, B. E.; PEREIRA, C. P. Macrófitos aquáticos do município de Rio Grande, Rio Grande do Sul, Brasil. Revista Agros, [s.n.], v. 20, n. 1/4, p. 45-52, 1985.

PEDRALLI, G.; MEYER, S. T.; TEIXEIRA, M. C.; STEHMANN, J. R. Levantamento dos macrófitos aquáticos e da mata ciliar do reservatório de Volta Grande, Minas Gerais, Brasil. Iheringia. Série Botânica, Porto Alegre, v. 43, p. 29-40, 1993.

PITELLI, R. A. Ecologia de várzeas. In: SIMPÓSIO NACIONAL SOBRE O APROVEITAMENTO DE VÁRZEAS, 1986, Jaboticabal. Anais... Jaboticabal: Fundação de Estudos e Pesquisas/UNESP, 1986. p. $15-24$.

POMPÊO, M. L. M.; MOSCHINI-CARLOS, V. Macrófitas aquáticas e perifiton: aspectos ecológicos e metodológicos. São Carlos: RIMA, 2003. 134 p.

RODRIGUES, R. R. Florestas ciliares? Uma discussão nomenclatural das formações ciliares. In: RODRIGUES, R. R.; LEITÃO FILHO, H. de F. (Eds.). Matas ciliares: conservação e recuperação. São Paulo: Editora da Universidade de São Paulo: FAPESP, 2000. p. 91-99.

ROSA, F. F. da; IRGANG, B. E. Comunidades vegetais de um segmento da planície de inundação do rio dos Sinos, Rio Grande do Sul, Brasil. Iheringia. Série Botânica, Porto Alegre, v. 50, p. 75-87, 1998.

SILVA, S. M.; BRITEZ, R. M. de; SOUZA, W. S. de; MOTTA, J. T. W. Levantamento florístico em área de várzea do rio Iguaçu, São Mateus do Sul - PR - Brasil. Brazilian Archives of Biology and Technology, Curitiba, v. 40, n. 4, p. 903-914, 1997.

SMITH, A. R.; PRYER, K. M.; SCHUETTPELZ, E.; KORALL, P.; SCHNEIDER, H.; WOLF, P. G. A classification for extant ferns. Taxon, Utrecht, v. 55, n. 3, p. 705-731, 2006.

SOUZA, M. C.; CISLINSKI, J.; ROMAGNOLO, M. B. Levantamento florístico. In: VAZZOLER, A. E. A. M.; AGOSTINHO, A. A.; HAHN, N.S. (Ed.). A planície de inundação do alto rio Paraná: aspectos físicos, biológicos e socioeconômicos. Maringá: EDUEM, 1997. p. 343-368.

THOMAZ, S. M.; BINI, L. M.; SOUZA, M. C. de; KITA, K. K.; CAMARGO, A. F. M. Aquatic macrophytes of Itaipu reservoir, Brazil: survey of species and ecological considerations. Brazilian Archives of Biology and Technology, Curitiba, v. 42, n. 1, p. 15-22, 1999.

TRYON, R. M.; TRYON, A. F. Ferns and allied plants. New York: Springer Verlag, 1982. 